\title{
Structural Investigations of Liquid-Crystalline Ethylcellulose
}

\author{
Yoshiyuki NishIO, Seiichi SUSUKI, and Toshisada TAKAHASHI \\ Faculty of Engineering, Fukui University, \\ Bunkyo 3-9-1, Fukui 910, Japan
}

(Received September 18, 1984)

\begin{abstract}
Solutions of ethylcellulose (EC) in acrylic acid (AA) form a mesomorphic phase with cholesteric nature in a limited range of concentrations and temperatures. Detailed phase behavior was investigated by optical techniques including polarized light microscopy, refractive index measurements, and spectrophotometry. Structural characterization was also performed for two kinds of solids prepared from the EC/AA liquid crystal: a polymer composite (EC/PAA) obtained by polymerizing the solvent AA and an EC film obtained by quiescently casting from the solution. Both solids possess optically anisotropic organization which comes from a mesomorphic state in the starting sample. It is shown by electron microscopy that there are many round particles 0.1 to $0.3 \mu \mathrm{m}$ in the EC/PAA composite, while in the EC cast film well-developed lamellar textures are formed by regular coalescence of round particles. Such morphological features are discussed in relation to the liquid crystalline structure in the initial solution.
\end{abstract}

KEY WORDS Ethylcellulose (EC) / Acrylic Acid (AA) / Cholesteric Liquid Crystal / Bulk Polymerization / EC/PAA Composite / EC Cast Film / Morphology /

In recent years, it has become apparent that a number of cellulose derivatives are capable of forming liquid-crystalline phases in highly concentrated solutions with appropriate solvents and sometimes in a fluid state without any solvent. ${ }^{1}$ Hydroxypropylcellulose (HPC) is the first reported liquid-crystalline polymer based on cellulose ${ }^{2}$ and the mesomorphic behavior of this polymer has been studied extensively by many workers. ${ }^{3-10}$ Since the finding of liquid-crystal formation in HPC, similar mesomorphic characterization has been described for a broad range of other cellulose derivatives including cellulose acetate, ${ }^{11,12}$ cellulose triacetate, ${ }^{5,12-16}$ cellulose acetatebutyrate, ${ }^{5,13}$ ethylcellulose,${ }^{5}$ acetoxypropylcellulose, ${ }^{17}$ trifluoroacetoxypropylcellulose, ${ }^{18}$ etc. ${ }^{1,19,20}$ and even for cellulose itself..$^{21,22}$ In most cases, these liquid crystals have a cholesteric nature, and often show iridescent colors in appearance under white light or swirl-like fingerprint patterns under a polarizing microscope, due to the periodic twisted arrangement of molecular orientation.

This paper is concerned with the structure and morphology of liquid-crystalline ethylcellulose (EC). It has already been noted by Bheda et $a l .{ }^{5}$ that this polymer forms anisotropic solutions with cholesteric order in acetic acid and dichloroacetic acid. Here we report that EC dissolves in a vinyl monomer, acrylic acid (AA), to form a cholesteric liquid-crystal phase at high polymer concentrations. The EC/AA liquid-crystalline solutions solidify not only into a bulky film by gradually evaporating AA but also into a polymer composite (EC/ PAA) by polymerizing AA with the aid of a radical initiator. Similar attempts to prepare a polymer composite have been made for a few liquid-crystalline systems of synthetic polypeptides in a vinyl monomer, ${ }^{23,24}$ where it is shown by optical microscopic observations 
that the initial mesophases are easily perpetuated within the resulting composites. In the preceding paper, ${ }^{10}$ we described, both by optical and electron microscopies, the morphological details of a polymer composite (HPC/PHEMA) prepared from a HPC/2hydroxyethyl methacrylate (HEMA) liquid crystal by bulk polymerization of HEMA.

The present work falls under the series of our recent investigations ${ }^{10,25-28}$ of liquidcrystalline polymers. In general, these polymers have a rigid or semi-rigid nature in molecular chains and hence even in the solid state exhibit distinct supermolecular structures and physical properties unlike common flexible polymers. Basic characterization of $\mathrm{EC} /$ AA solutions is carried out using optical techniques; polarized optical microscopy, measurements of refractive index, and UV spectrophotometry. Morphological features of the EC/ PAA composite and EC cast film are examined by means of polaried-light and electron microscopies.

\section{EXPERIMENTAL}

\section{Sample Preparation}

Ethylcellulose (EC) purchased from Tokyo Kasei Kogyo Co. was used in this study. The nominal value of the viscosity of a $5 \%$ solution of this polymer in toluene-ethanol $(80: 20)$ was 20 centipoise at $25^{\circ} \mathrm{C}$. Acrylic acid (AA), $\alpha, \alpha^{\prime}$ azobisisobutyronitrile (AIBN), and ethylene dimethacrylate (EDM) were reagent-grade chemicals and used without further purification.

EC/AA solutions were prepared at polymer concentrations ranging from 25 to $57 \mathrm{wt} \%$ by mixing weighed $\mathrm{EC}$ and $\mathrm{AA}$ at room temperature.

To a portion of $50 \mathrm{wt} \% \mathrm{EC} / \mathrm{AA}$ solution, which gave the cholesteric mesophase shown below, AIBN (radical initiator for polymerization) and EDM (crosslinking agent) were added at concentrations of about 2 and $3 \mathrm{wt} \%$, respectively, with respect to the amount of
AA. The solution was charged to a polyethylene capsule in the shape of cylinder, $8 \mathrm{~mm}$ in diameter and $15 \mathrm{~mm}$ in height. The capsule was then stored in a dark place at $18^{\circ} \mathrm{C}$ for a week prior to initiating polymerization, to allow the liquid crystalline phase to develop well in the solution. Polymerization of the sample at $40 \pm 3^{\circ} \mathrm{C}$ for 3 days in a thermostat yielded a bulk composite comprizing EC and crosslinked poly(acrylic acid) (PAA).

A solid film of EC ( $c a .1 .5 \mathrm{~mm}$ thick) was prepared from a $50 \mathrm{wt} \%$ EC/AA solution sufficiently aged to form a liquid crystal, by the gradual evaporation of AA over a period of 3 weeks at room temperature.

\section{Measurements}

Measurements for characterizing EC/AA solutions included refractive index measurements, polarized light microscopy, and spectrophotometry (UV absorption). Refractive indices were measured using an Abbé refractometer with a rotatable polarizer mounted over the eyepiece. An Olympus polarizing microscope was used to observe anisotropic phases in the solutions. For the observations, samples were sealed into a thin layer of solution between a slide and cover glass. Absorption spectra were measured to detect the selective reflection band due to a cholesteric structure in liquid crystals. The measurements were carried out on highly concentrated solutions sandwiched between parallel glass plates separated by a Teflon spacer of $200 \mu \mathrm{m}$ thickness, using a Shimadzu UV200S spectrophotometer.

Morphological studies of the EC/PAA composite and EC cast film were performed by polarized-light and electron microscopies. Thin sections cut from these solids were used for optical microscopic observations. To elucidate the internal supermolecular architecture of the solids, fracture-surface morphologies were observed with a JEOL 200B electron microscope. For this purpose, the solids were fractured in the frozen state in liquid nitro- 
gen and the fragments were examined by the following replication techniques:

The composite specimens were placed on a highly viscous aqueous solution of poly(vinyl alcohol) (PVA) spread on a glass plate, and then detached from the solidified PVA film with a pair of tweezers in chloroform under ultrasonic irradiation. The remaining PVA film was supplied for preparation of the replica membrane by shadowing with $\mathrm{Au} / \mathrm{Pd}$ alloy and coating with carbon. The PVA substrate was ultimately removed from the replica membrane by dissolution with water on a copper grid. On the other hand, replica membranes of fragments of the EC cast film were obtained by directly coating the fracture-surfaces with $\mathrm{Au} / \mathrm{Pd}$ and carbon and dissolving $\mathrm{EC}$ in chloroform.

\section{RESULTS AND DISCUSSION}

\section{Phase Study of EC/AA Solutions}

Optical measurements of EC/AA solutions were carried out after standing for at least a month.

Values of the refractive indices of EC/AA solutions are listed in Table I, where the visual appearance of the solutions and time required for $\mathrm{EC}$ to dissolve in $\mathrm{AA}$ at each concentration are also summarized. The values of the indices were obtained with the Sodium-D line at room temperature. At EC concentrations below 40 $\mathrm{wt} \%$, the solutions gave a single line in the viewing telescope of the refractometer, when spread between the prism surfaces. At higher concentrations, however, they gave two distinguishable lines attributed to the principal refractive indices parallel $\left(n_{\|}\right)$and perpendicular $\left(n_{\perp}\right)$ to the plane of the prism surfaces. Thus, EC/AA solutions are optically isotropic at concentrations lower than $40 \mathrm{wt} \%$, but birefringent above this concentration.

The EC/AA anisotropic samples were generally cloudy. Especially above $45 \mathrm{wt} \%$, the higher the concentration, the greater the turbidity of the solutions. In the concentration range 40 to $55 \mathrm{wt}^{\mathrm{o}} \%$, the solutions showed iridescent colors which varied as a function of concentration. At $40 \mathrm{wt} \%$, the solution exhibited colors from red to orange, and the range moved to the violet end of the spectrum at 55 $\mathrm{wt} \%$, as shown in Table I. The $57 \mathrm{wt} \%$ solution showed no iridescent color and was somewhat gelatinous.

Results of the spectrophotometric measurements on three anisotropic solutions are given in Figure 1, which shows evidence of a cholesteric mesophase in the solutions. The spectra

Table I. Basic data of EC/AA solutions investigated

\begin{tabular}{|c|c|c|c|c|c|c|}
\hline \multirow{2}{*}{$\frac{\text { Concentration }}{w^{\circ} \%}$} & \multicolumn{2}{|c|}{ Refractive indices } & \multirow{2}{*}{$\begin{array}{l}\text { Birefringence } \\
\begin{array}{c}\Delta n\left(=n_{\|}-n_{\perp}\right) \\
\quad \times 10^{4}\end{array}\end{array}$} & \multirow{2}{*}{$\begin{array}{l}\text { Dissolving } \\
\text { time/day }\end{array}$} & \multirow{2}{*}{\multicolumn{2}{|c|}{ Visual appearance }} \\
\hline & $n_{\|}$ & $n_{\perp}$ & & & & \\
\hline 25 & 1.4375 & - & 0 & 2 & \multirow{2}{*}{\multicolumn{2}{|c|}{ Transparent }} \\
\hline 30 & 1.4402 & - & 0 & 3 & & \\
\hline 40 & 1.4468 & 1.4462 & 6 & 6 & \multicolumn{2}{|c|}{$\begin{array}{l}\text { Translucent, faint } \\
\text { iridescent (red-orange) }\end{array}$} \\
\hline 45 & 1.4494 & 1.4484 & 10 & 8 & & (Red-green) \\
\hline 50 & 1.4522 & 1.4509 & 13 & 9 & $\begin{array}{l}\text { Fairly turbid, } \\
\text { iridescent }\end{array}$ & (Green-violet) \\
\hline 55 & 1.4552 & 1.4529 & 23 & 12 & & (Pale violet) \\
\hline 57 & 1.4566 & 1.4543 & 23 & $>14$ & \multicolumn{2}{|l|}{$\begin{array}{l}\text { Very turbid, } \\
\text { noniridescent, } \\
\text { gelatinous }\end{array}$} \\
\hline
\end{tabular}




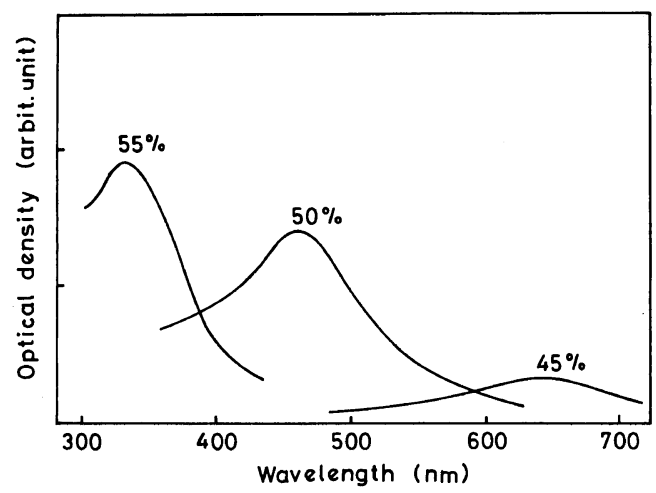

Figure 1. UV spectra of EC/AA anisotropic solutions, showing selective reflection bands due to a cholesteric twisted structure.

were determined by measuring the optical absorbance of light transmitted normal to the sample sandwiched between parallel glass plates. It may be assumed that the curves represent selective reflection bands due to a periodic twisted structure of cholesteric liquid crystals, and not extinction bands due to intrinsic light-absorption of EC and/or AA. The band peak becomes sharper and shifts to the blue side in order of the 45, 50, and $55 \mathrm{wt} \%$ solutions. This is attributable to development of the cholesteric order into a more uniform and stronger state accompanying a decrease in periodicity pitch. With the wavelength $\left(\lambda_{\mathrm{M}}\right)$ at the maximum reflection and the average refractive index $\left(\tilde{n}=\left(2 n_{\|}+n_{\perp}\right) / 3\right)$ of each solution, the cholesteric pitch $(P)$ was estimated from the de Vries equation, ${ }^{29} \lambda_{\mathrm{M}}=\tilde{n} P$, applicable to the reflection of light incident normally on a planar cholesteric sample. The evaluated pitches were $440 \mathrm{~nm}(45 \mathrm{wt} \%), 320 \mathrm{~nm}(50$ $\mathrm{wt} \%$ ), and $230 \mathrm{~nm}\left(55 \mathrm{wt}^{\mathrm{o}} \%\right.$ ), at which values, the pitch varies inversely as approximately the third power of the weight fraction of EC. A similar dependence of pitch on concentration has been observed in HPC/water ${ }^{4}$ and acetoxypropylcellulose/acetone ${ }^{17}$ systems.

Further characterization of the phase behavior of EC/AA solutions was carried out

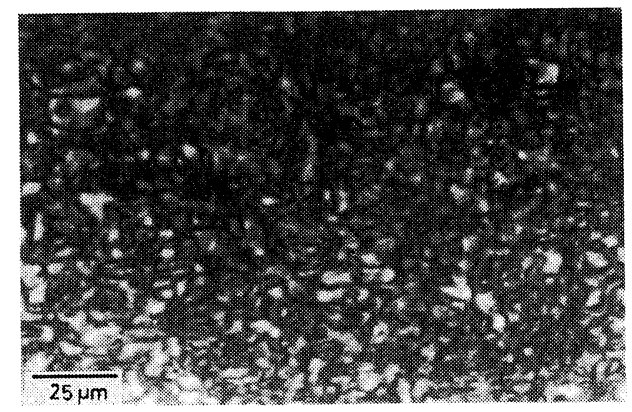

Figure 2. Polarized optical micrograph of a $55 \mathrm{wt} \%$ EC/AA solution.

with a polarizing microscope. The solutions passed no light between crossed polars until an EC concentration of $40 \mathrm{wt} \%$. The 40 and 45 $\mathrm{wt} \%$ solutions indicated biphasic structures consisting of a mixture of anisotropic phases with grid-like patterns and dark isotropic areas. Such samples may thus have embryonic cholesteric orders. More concentrated solutions gave single-phase anisotropic structures of mosaic texture of somewhat distorted, crossed striations, as illustrated for the $55 \mathrm{wt} \%$ sample in Figure 2. This distinctive morphology seems essentially the same as that reported earlier for the HPC/water liquid crystal. $^{2,4}$ At present, there is no complete explanation for the mechanism of formation of such a texture. Shimamura has recently discussed a similar birefringent structure preserved in a HPC film crystallized from the concentrated aqueous solution. ${ }^{8} \mathrm{~A}$ detailed characterization of this kind of structure observed in cellulosebased liquid crystalline systems will be presented by the authors in the near future.

The temperature dependence of the anisotropic phase in EC/AA solutions was examined on a hot stage of the polarizing microscope. A phase diagram of this system is shown in Figure 3. The temperature at which an anisotropic solution completely transformed into an isotropic liquid when heated from room temperature at a rate of $1 \mathrm{~K} \mathrm{~min}^{-1}$ is plotted against the EC concentration for several samples. The critical temperature of 


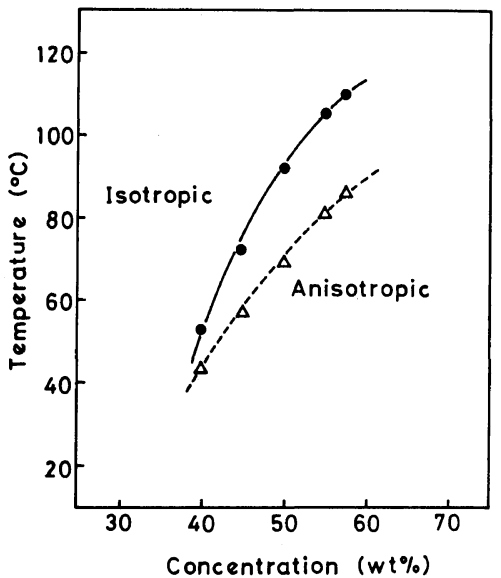

Figure 3. Phase diagram of the EC/AA system, determined by polarized light microscopy: $\bigcirc$, temperature of the perfect transition from anisotropic to isotropic phase; $\triangle$, critical temperature of isotropic phases emergence on heating.

emergence of isotropic phases on heating is also plotted in this figure. Below this temperature, each EC/AA solution remains relatively stable with the starting anisotropic phase. For example, when the $50 \mathrm{wt} \%$ sample was warmed, the solution remained uniformly anisotropic up to $70^{\circ} \mathrm{C}$, in spite of gradual disturbance of the initial grid-like texture above $c a$. $55^{\circ} \mathrm{C}$. At temperatures higher than $70^{\circ} \mathrm{C}$, dark, isotropic areas appeared in the solution and greatly increased with increasing temperature. Ultimately, the perfect transition from anisotropic to isotropic phase was observed at $92^{\circ} \mathrm{C}$. In the present system, no significant formation of a white gel or precipitation such as observed in HPC in water ${ }^{3}$ took place at elevated temperatures irrespective of EC concentration.

\section{Morphological Study of EC/PAA Composite}

If an EC/AA solution is solidified by polymerizing the solvent in a proper concentration and temperature range where the sample has a cholesteric mesophase, the resulting polymer composite may possess anisotropic organization reflecting the initial liquid-crystalline structure. Acting on this possibility, an EC/

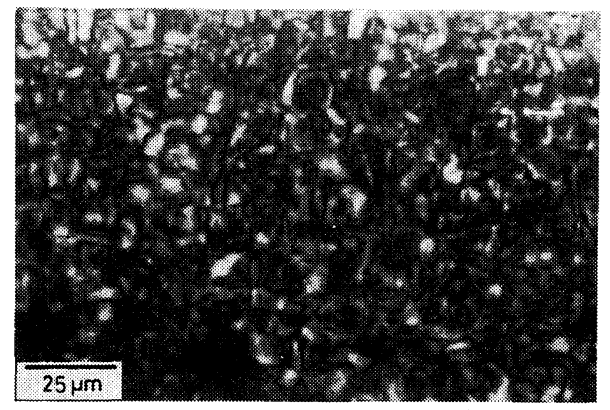

Figure 4. Polarized optical micrograph of an EC/PAA composite prepared from a $50 \mathrm{wt} \% \mathrm{EC} / \mathrm{AA}$ solution by polymerizing AA.

PAA composite was prepared from a $50 \mathrm{wt} \%$ EC/AA iridescent solution containing a small amount of radical initiator (AIBN) and crosslinking agent (EDM), by bulk polymerization at about $40^{\circ} \mathrm{C}$ for 3 days.

The composite thus obtained displayed no iridescent color and was visually quite turbid. It is undeniable that some change in cholesteric nature occurred in this system during polymerization. We recognized qualitatively that the present polymerization caused the system to contract slightly in volume. This effect presumably gave rise to local disturbance of the cholesteric structure and a decrease in pitch, resulting in vanishing of the iridescent colors.

Under a polarizing microscope, however, the composite had anisotropic organization similar to that observed in the starting liquid crystal, as seen in Figure 4. Accordingly, it is likely that the structure which developed in the original mesomorphic state was considerably preserved in this product, even after the solvent $\mathrm{AA}$ was polymerized and crosslinked.

For elucidation of the internal structure of the product, electron microscopic observations were carried out on fragments obtained by fracturing it in liquid nitrogen. For the sample preparations, composite specimens were forcefully peeled off the poly(vinyl alcohol) substrate for replication in chloroform under ultrasonic irradiation. The EC and residual AA 


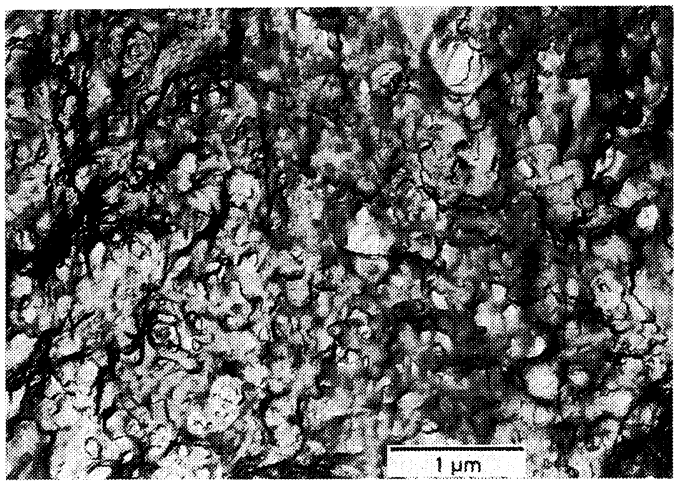

Figure 5. Electron micrograph of an EC/PAA composite.

monomer are probably extracted from the composite, whereas crosslinked PAA partially remains on the substrate without being completely detached from the surface. Figure 5 shows a representative electron micrograph revealing well the fracture-surface morphology of the EC/PAA composite. There are numerous traces of round particles $0.1-0.3 \mu \mathrm{m}$ in diameter surrounded by somewhat complicated textures. These textures may possibly correspond to a network system of crosslinked PAA, constructed in the liquid-crystalline state of the EC/AA solution. It is thus plausible that the ordered regions of EC are perpetuated as vestiges of round particles in such a polymer network after the mesogens are eliminated from the system.

Similar round particles were found in a HPC/PHEMA composite previously studied, ${ }^{10}$ and have actually been shown to be formed by parallel stacking of thin disks. We cannot conclude immediately here that such a round particle is identified with a cholesteric domain having a layered feature. At least, however, it appears more likely that this kind of particle behaves as a common fundamental structural unit in the formation of supermolecular architectures of liquid-crystalline cellulose derivatives, when their solids are prepared from the cholesteric ordered state. This is also emphasized below for an EC cast film.

\section{Morphological Study of Quiescent Cast EC Film}

The gradual evaporation of AA over a period of 3 weeks at room temperature allowed an EC/AA liquid-crystalline solution $(50 \mathrm{wt} \%)$ to be made into a solid film of EC. The film displayed optical anisotropy easily detectable with a polarizing microscope. The image viewed between crossed polars was similar to that of the EC/PAA composite, except that the birefringent phase had a more closely striated pattern of dark distorted crosses. It is also remarkable that, when an isotropic EC/AA solution $(5 \mathrm{wt} \%$ ) was evaporated to dryness at room temperature over a period of 10 days, the resulting film showed no distinguishing birefringent region between the crossed polars. This suggests that the structural character in the solid state of liquid-crystalline polymers is intimately related to the structure matured sufficiently in the mesomorphic state.

The EC film cast from the liquid crystal exhibited very interesting morphologies under electron microscope. The fracture-surface morphologies are shown in Figure 6. Figure 6 a shows a well-developed lamellar structure extending over the whole area, with strata made in a series of steps. An enlarged photograph of a part of Figure 6a is given in Figure $6 \mathrm{~b}$, from which it can readily be seen that each lamella was formed as a result of coalescence of round particles with their neighbors. In another electron micrograph (Figure 6c) taken of a different area of the same sample, even illdefined round particles are no longer present; these particles seem to regularly develop into united bodies as lamellae by successive coalescence between neighboring particles. It is supposed that an abundance of such lamellar textures in the EC film is produced by some mechanical effect giving rise to destruction of the initial mesomorphic structure in which presumably discrete round particles are densely packed. 

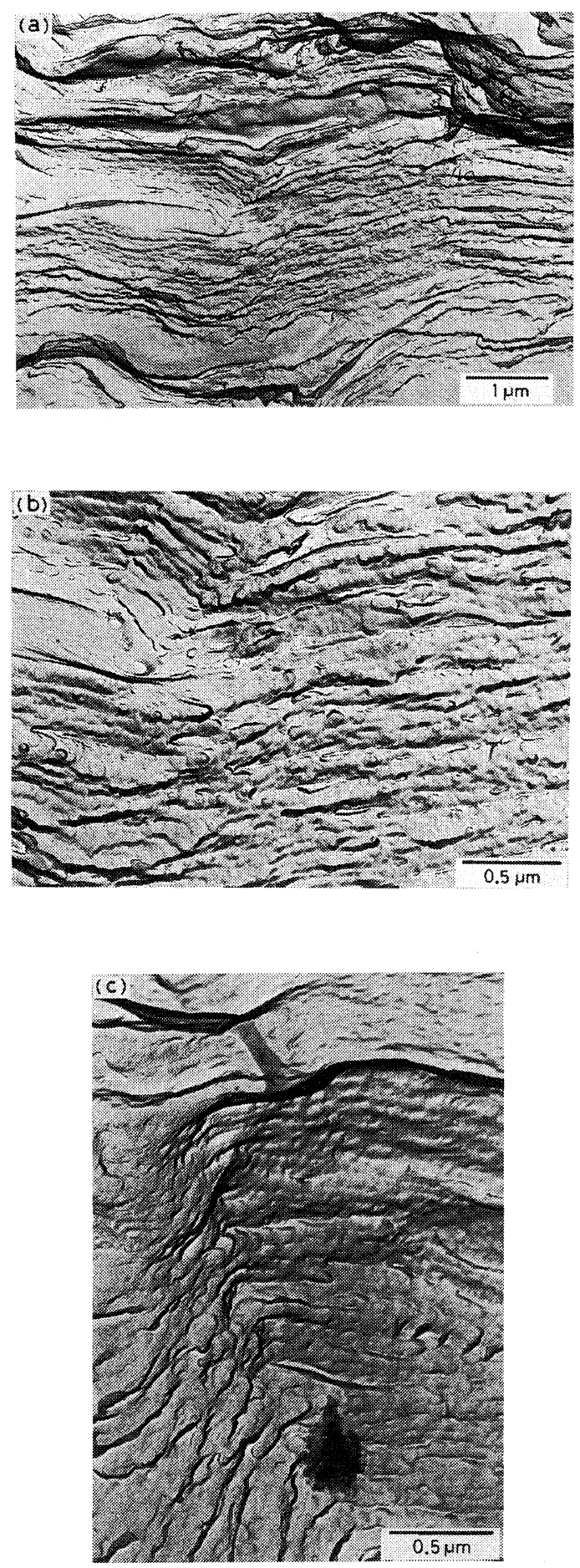

Figure 6. Electron micrographs of an EC film prepared by casting from an EC/AA liquid-crystalline solution. (b) is an enlarged part of the micrograph (a).

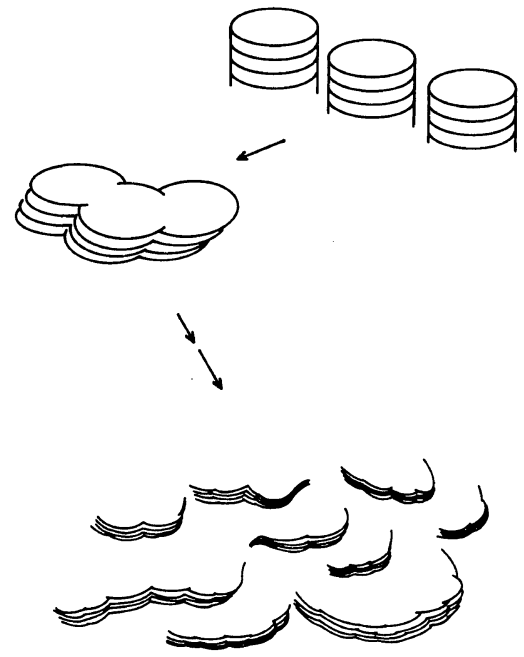

Figure 7. Schematic illustration showing the development of a lamellar structure by repetition of the coalescence of adjacent round particles.

The round particle mentioned here can be regarded as substantially the same as that described in the preceding section. Moreover, it may be taken that the particle is composed of parallel-stacked disks, from analogy with the structural aspect of HPC solids studied earlier. ${ }^{10}$ Based on such reasonable assumptions, the morphological features of the EC cast film can be interpreted as follows: When an EC film is cast from the EC/AA liquid crystal, the system inevitably shrinks in the course of solidification more strongly than in the case of polymerization of the same system, since the former process is accompanied by evaporation of the solvent. Due to continuous strain induced within the liquid-crystalline system by shrinking, the constituent round particles deform easily with slippage of the layered disks and then grow into a large united body by lateral coalescence with their neighbors, as schematically shown in Figure 7. Repetition of similar coalescence behavior makes the united body increasingly larger, resulting in the formation of a tightly associated lamellar structure. 


\section{CONCLUSIONS}

Structural characterization of ethylcellulose (EC) was performed not only for solutions in acrylic acid (AA) but also for solids prepared from the liquid crystal. Our conclusions are as follows:

1) The EC/AA solution forms a liquidcrystal phase of an apparently cholesteric nature at high EC concentrations. A precise phase diagram of this system was determined by examining the concentration and temperature dependences of the optical anisotropy.

2) An EC/PAA composite can be prepared easily from the EC/AA liquid crystal by bulk polymerization of AA. The polymer composite retained well the starting mesomorphic organization, although the iridescent cholesteric colors were lost. Electron microscopy showed many round particles to be present in this composite and surrounded by complicated textures presumably corresponding to a network system of crosslinked PAA. This study provides additional information on the design of multicomponent polymer blends having specific supermolecular structures.

3) An EC film obtained by casting from the EC/AA liquid-crystalline solution also possesses an optically anisotropic structure which comes from the starting mesomorphic state. Under the electron microscope, this sample exhibited lamellar structures which developed by repetition of the coalescence of constituent round particles through contractional strain induced during solidification.

\section{REFERENCES}

1. D. G. Gray, J. Appl. Polym. Sci., Appl. Polym. Symp., 37, 179 (1983).

2. R. S. Werbowyj and D. G. Gray, Mol. Cryst. Liq. Cryst. Lett., 34, 97 (1976).
3. R. S. Werbowyj and D. G. Gray, Macromolecules, 13, 69 (1980).

4. Y. Onogi, J. L. White, and J. F. Fellers, J. Polym. Sci., Polym. Phys. Ed., 18, 663 (1980).

5. J. Bheda, J. F. Fellers, and J. L. White, Colloid Polym. Sci., 258, 1335 (1980).

6. T. Tsutsui and R. Tanaka, Polym. J., 12, 473 (1980).

7. T. Asada, K. Toda, and S. Onogi, Mol. Cryst. Liq. Cryst., 68, 231 (1981).

8. K. Shimamura, Makromol. Chem. Rapid Commun., 4, 107 (1983).

9. K. Shimamura, J. L. White, and J. F. Fellers, J. Appl. Polym. Sci., 26, 2165 (1981).

10. Y. Nishio, T. Yamane, and T. Takahashi, J. Polym. Sci., Polym. Phys. Ed., in press.

11. S. M. Aharoni, Mol. Cryst. Liq. Cryst. Lett., 56, 237 (1980).

12. P. Navard, J. M. Haudin, S. Dayan, and P. Sixou, J. Polym. Sci., Polym. Lett. Ed., 19, 379 (1981).

13. J. Bheda, J. F. Fellers, and J. L. White, J. Appl. Polym. Sci., 26, 3955 (1981).

14. D. L. Patel and R. D. Gilbert, J. Polym. Sci., Polym. Phys. Ed., 19, 1449 (1981).

15. S. Dayan, P. Maissa, M. J. Vellutini, and P. Sixou, J. Polym. Sci., Polym. Lett. Ed., 20, 33 (1982).

16. G. H. Meeten and P. Navard, Polymer, 23, 1727 (1982).

17. S.-L. Tseng, A. Valente, and D. G. Gray, Macromolecules, 14, 715 (1981).

18. S. M. Aharoni, J. Polym. Sci., Polym. Lett. Ed., 19, 495 (1981).

19. P. A. Patton and R. D. Gilbert, J. Polym. Sci., Polym. Phys. Ed., 21, 515 (1983).

20. P. Zugenmaier, J. Appl. Polym. Sci., Appl. Polym. Symp., 37, 223 (1983).

21. H. Chanzy, A. Peguy, S. Chaunis, and P. Monzie, $J$. Polym. Sci., Polym. Phys. Ed., 18, 1137 (1980).

22. D. L. Patel and R. D. Gilbert, J. Polym. Sci., Polym. Phys. Ed., 19, 1231 (1981).

23. T. Tsutsui and T. Tanaka, J. Polym. Sci., Polym. Lett. Ed., 15, 475 (1977).

24. T. Tsutsui and R. Tanaka, Polymer, 22, 117 (1981).

25. T. Takahashi, H. Iwamoto, K. Inoue, and I. Tsujimoto, J. Polym. Sci., Polym. Phys. Ed., 17, 115 (1979).

26. T. Takahashi, Y. Yamamoto, and I. Tsujimoto, $J$. Macromol. Sci.-Phys., B16, 539 (1979).

27. T. Takahashi, M. Miura, and K. Sakurai, J. Appl. Polym. Sci., 28, 579 (1983).

28. Y. Nishio and T. Takahashi, J. Macromol. Sci.Phys., to be published.

29. H. de Vries, Acta Cryst., 4, 219 (1951). 